\title{
Frequency response of Michelson- and Sagnac-based interferometers
}

\author{
J. Mizuno ', A. Rüdiger, R. Schilling, W. Winkler, K. Danzmann ${ }^{2}$ \\ Max-Planck-Institut für Quantenoptik, Hans-Kopfermann-Str. I, 85748 Garching, Germany
}

Received 5 November 1996; revised 18 January 1997; accepted 24 January 1997

\begin{abstract}
The frequency response of Michelson- and Sagnac-based interferometers are evaluated and compared, considering the application for gravitational-wave detection. It is shown that Sagnac-based interferometers have some interesting features, but in general do not offer advantages over Michelson-based ones for the detection of gravitational waves.

PACS: 04.80.Nn; 07.60.Ly; 42.25.Hz; 95.55.Ym

Keywords: Michelson interferometer; Sagnac interferometer; Power recycling; Signal recycling; Resonant sideband extraction; Gravitational wave detector
\end{abstract}

\section{Introduction}

Precise position measurements using interferometry often adopt a configuration based on the Michelson interferometer. This is, however, not the only possibility of making two-beam interferometers; there are also other choices, such as the Sagnac interferometer.

Among the most sensitive interferometers currently developed are those for gravitational-wave detection [1-3]. In this field, several modifications of the basic Michelson interferometer have been proposed in order to achieve the extremely high phase sensitivity required $\left(\sim 10^{-9} \mathrm{rad}\right)$. These include the use of multiple reflections [4] or optical cavities [5] in the arms, power recycling [5], signal recycling [6], and resonant sideband extraction [7,8].

Recently, making such a gravitational-wave detector based on the Sagnac interferometer is proposed again as an alternative approach (see Ref. [9] and references therein).

\footnotetext{
'E-mail: mizuno@mpq.mpg.de.

Also at: Universität Hannover, Appelstr. 2, 30167 Hannover. Germany.
}

So far, however, its behavior is not investigated as thoroughly as that of the Michelson one. In this paper, we discuss the feasibility of Sagnac-based interferometers, with particular attention paid to applying the enhancement schemes that are proposed for Michelson-based interferometers.

\section{Optical measurement of gravitational waves}

Suppose a gravitational wave propagates along the $z$-axis, whose polarization axes coincide with the $x$ - and $y$-axes. Then the light (of angular frequency $\omega_{0}$ ) traveling through an optical path of length $L$ along the $x$-axis acquires a phase delay $[10,8]$

$\phi(t)=\frac{\omega_{0} L}{c}-\frac{\omega_{0}}{2} \int_{t-L / c}^{t} h\left(t^{\prime}\right) \mathrm{d} t^{\prime}+\mathrm{O}\left(h^{2}\right)$.

Here the strain amplitude $h$ of the gravitational wave is expected to be extremely small $\left(|h| \ll 10^{-18}\right)$ and thus higher order effects can be ignored. The second, time-varying term (which we refer to as the phase shift $\delta \phi$ ) represents the effect of the gravitational wave. 
For light traveling along the $y$-axis, the polarity of the phase shift is opposite due to the quadrupole nature of the gravitational waves. The differential phase shift between the two orthogonal arms will be measured interferometrically.

Each Fourier component of the phase shift, with an angular frequency $\omega_{\mathrm{g}}$, can be considered as a phase modulation that produces upper and lower sidebands at $\omega_{0} \pm \omega_{\mathrm{g}}$. Amplitude and phase of each sideband are determined by the optical configuration. They are represented by the optical response $G(\omega)$, where $\omega$ is the frequency offset from the carrier, i.e. $\pm \omega_{\mathrm{g}}$ for the upper and lower sidebands, respectively.

The sum of these two sidebands is the signal we finally extract from an interferometer $[8,11]$. In order to compare the best sensitivities achievable using various kinds of interferometers, the sideband amplitudes at the output for given input light power must be compared. (It is not necessarily true that one can always achieve this optimum sensitivity; but this is an issue different from the comparison of optical configurations done here.)

The normalized response $F$ is defined as the transfer function from gravitational wave amplitude $h$ to the sum of upper- and lower-sideband amplitudes normalized by the injected light amplitude $\left(\alpha \sqrt{P_{0}}\right)$ :

$$
F\left(\omega_{\mathrm{g}}\right)=G_{\text {int }}\left(\omega_{\mathrm{g}}\right)+G_{\text {int }}^{*}\left(-\omega_{\mathrm{g}}\right)
$$

where ${ }^{*}$ denotes the complex conjugate. (This definition assumes that the phase-modulation component is detected, which is the best scheme for broad-band responses and is commonly used. See Refs. [8,11] for more detail.)

In the following discussion, we assume the shot noise to be the limiting noise source in the frequency range of interest. Then, by using this normalized response, the sensitivity of an interferometric detector to gravitational waves is represented as $[8,12]$

$\tilde{h}\left(\omega_{\mathrm{g}} / 2 \pi\right)=\frac{1}{2\left|F\left(\omega_{\mathrm{g}}\right)\right|} \sqrt{\frac{2 \hbar \omega_{0}}{\eta P_{0}}}$,

or by the normalized scale used in the figures (see the caption of Fig. 1),

$$
\begin{aligned}
\tilde{h}\left(\omega_{\mathrm{g}} / 2 \pi\right) \approx & \frac{1.1 \times 10^{-20} / \sqrt{\mathrm{Hz}}}{\text { normalized gain }} \\
& \times\left[\frac{l}{3 \mathrm{~km}}\right]^{-1}\left[\frac{\lambda}{1 \mu \mathrm{m}}\right]^{1 / 2}\left[\frac{\eta P_{0}}{10 \mathrm{~W}}\right]^{-1 / 2} .
\end{aligned}
$$

Here $\tilde{h}$ represents the linear spectral density of the gravitational-wave amplitude that gives unity signal-to-noise ratio, and $\eta$ is the efficiency of the photo-detector.

The time domain formula in Eq. (1) is converted to the frequency domain hy taking the Laplace transform of each side:

$$
\begin{aligned}
\mathscr{L}\{ & \delta \phi(t)\} \\
& =\frac{\omega_{0}}{2} \mathscr{L}\left\{\int_{t-L / c}^{t} h\left(t^{\prime}\right) \mathrm{d} t^{\prime}\right\} \\
& =\frac{\omega_{0}}{2}\left[\mathscr{L}\left\{\int_{0}^{t} h\left(t^{\prime}\right) \mathrm{d} t^{\prime}\right\}-\mathscr{L}\left\{\int_{0}^{t-L / c} h\left(t^{\prime}\right) \mathrm{d} t^{\prime}\right\}\right] \\
& =\frac{\omega_{0}}{2} \frac{1-\mathrm{e}^{-s L / c}}{s} \mathscr{L}\{h(t)\} .
\end{aligned}
$$

The transfer function $X$ from gravitational wave amplitude $h$ to phase shift $\delta \phi$ is defined as the ratio of their Laplace transforms, thus

$$
\underset{h \rightarrow \delta \phi}{X}=\frac{\mathscr{L}\{\delta \phi(t)\}}{\mathscr{L}\{h(t)\}}=\frac{\omega_{0}}{2} \frac{1-\mathrm{e}^{-s L / c}}{s} .
$$

(In order to obtain the frequency response, the Laplace parameter $s$ must be replaced by $\mathrm{i} \omega$.)

The optical response $G$ is the sideband amplitude relative to the injected carrier amplitude $a_{0} \propto \sqrt{P_{0}}$. Each (upper or lower) sideband produced by a (weak) phase modulation with an index $\delta \phi$ has an amplitude of $a_{0} J_{1}(\delta \phi) \simeq a_{0} \delta \phi / 2$ where $J_{1}$ is the first order Bessel function. Thus, the optical response $G_{\text {arm }}$ for one arm of optical path length $L$ can be written as

$G_{\text {arn }}=\rho_{\text {arm }} \frac{\omega_{0}}{4} \frac{1 \mathrm{e}^{-s L / c}}{s}$.

Here $\rho_{\text {arm }} \leq 1$ represents the amplitude attenuation factor during the round trip in the arm.

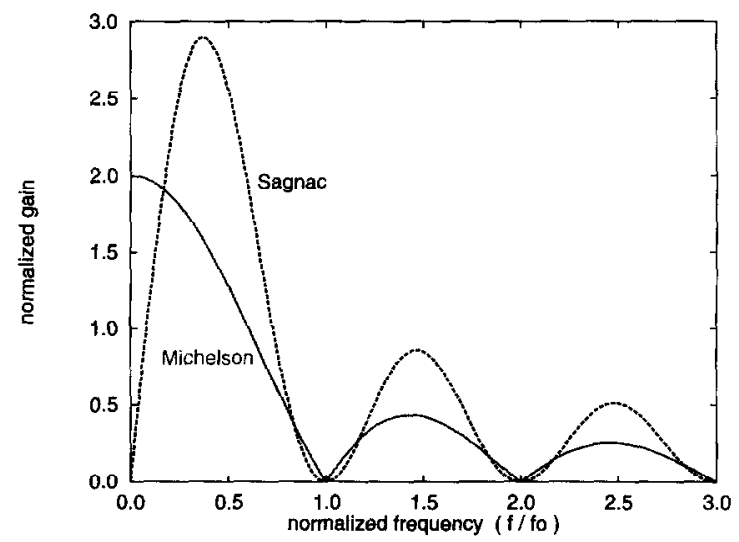

Fig. 1. Response of Michelson and Sagnac interferometers, both having a single bounce in each arm. The vertical scale is the gain $|F|$, normalized so that the DC gain of a (non-power-recycled) Michelson interferometer with $N$ transits in one arm will be $N$. (Note that $N=2$ for a single bounce case. See Eq. (4) to convert this to the sensitivity.) The horizontal scale is the frequency normalized by $f_{0}=c / 2 l \approx 50 \mathrm{kHz} \times[l / 3 \mathrm{~km}]^{-1}$. The same scales are used in the following frequency response figures (except for the horizontal scale of Fig. 11). 
Note that the magnitude of $G_{u r m}$ at DC increases proportional to the optical path length $L$ :

$G_{\text {arm }} \rightarrow \rho_{\text {arm }} \frac{\omega_{0}}{4} \frac{L}{c} \quad$ as $s \rightarrow 0$.

Thus, a long optical path is desirable for our purpose. How to realize it is discussed in the following sections.

\section{Multiple reflections in the arms}

For purely differential phase shifts, the effects on the light in the two arms of an interferometer have the same amplitude but opposite polarity. Thus the optical response of a simple (i.e. single bounce) Michelson interferometer with the optical path length $L$ is the same as that for one arm given in Eq. (7), ignoring the constant factor that comes from the existence of the two arms. (This factor is included in the definition of Eq. (3).) Note that the optical path length $L$ for one arm of a Michelson interferometer is twice the armlength $l$, i.e. $L=2 l$.

In the case of a Sagnac interferometer with the same path length $L$ in one arm, the light exiting from one arm will experience an additional round trip in the other arm. Since we are assuming a very weak modulation, we can neglect higher order effects, i.e. the modulation of the sidebands. Thus, the total amount of a single sideband is the sum of that produced in the first arm and that in the second arm.

The carrier entering the second arm has experienced a finite loss in the first arm, which is represented by the amplitude attenuation factor $\rho_{\mathrm{arm}}$. On the other hand, the sideband produced in the first arm experiences loss and phase shift in the second arm. Considering these effects, the optical response of the Sagnac interferometer to gravitational waves can be written as

$$
\begin{aligned}
G_{\text {Sag }} & =\rho_{\text {arm }}\left(1-\mathrm{e}^{-s L / c}\right) G_{\text {arm }} \\
& =\rho_{\mathrm{arm}}^{2} \frac{\omega_{0}}{4} \frac{\left(1-\mathrm{e}^{-s L / c}\right)^{2}}{s} .
\end{aligned}
$$

Fig. 1 shows the gain $|F|$, the magnitude of the normalized responses, of a Michelson and of a Sagnac interferometer, assuming that both have the same optical path length $L$ in one arm. Both responses have zeros (of order 1 in the Michelson and of order 2 in the Sagnac case) at multiples of $f_{z}=c / L$. The Michelson response has its peak at $\mathrm{DC}$, whereas the Sagnac one has a zero at DC and the peak gain at $\sim 0.371 f_{\mathrm{z}}$. The peak gain of the Sagnac response is about 1.45 times higher than that of the Michelson. The bandwidths ${ }^{3}$ measured at the $-3 \mathrm{~dB}$ points are $0.443 f_{\mathrm{z}}$

\footnotetext{
${ }^{3}$ Here we measure strictly at $-3 \mathrm{~dB}$ of the gain at the peak for an accurate comparison, although $0.5 f_{\mathrm{z}}$ is more commonly used for the bandwidth of a Michelson. The Sagnac bandwidth is shown in two numbers that represent the upper and lower cut-off frequencies, respectively.
}
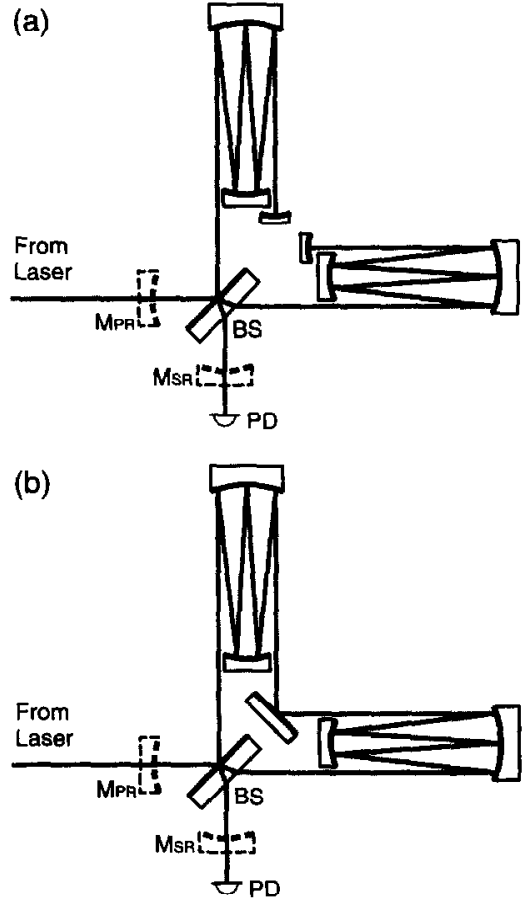

Fig. 2. Schematics of (a) Michelson and (b) Sagnac layout using multiple reflections in the arms. Positions for possible power- and signal-recycling mirrors $\left(\mathrm{M}_{\mathrm{PR}}, \mathrm{M}_{\mathrm{SR}}\right)$ are also shown (in dashed lines).

for the Michelson and $(0.581-0.182) f_{z}=0.399 f_{z}$ for the Sagnac.

As mentioned earlier, a long optical path is desirable for our purpose. The armlength is, however, limited by practical considerations such as cost, at least for groundbased detectors. In order to realize a longer path length $L$ within a given armlength $l$, the use of multiple reflections in an arm (as shown in Fig. 2) was proposed [4] and is commonly adopted. This does not change the characteristic shape of the frequency response, but alters the scaling of both axes: the overall height increases by $b=N / 2$ where $N$ is the number of transits in one arm, and the frequency scale decreases by the same factor, i.e. the first zero $f_{z}$ becomes $c / N l$ instead of $c / 2 l\left(=f_{0}\right.$ in the figures $)$.

There is, however, a limitation on the optical path length achievable due to the finite size of the beam. The Gaussian radius of the beam at the mirror in the symmetric confocal case is given by

$w=\sqrt{l \lambda / \pi}$

where $\lambda=2 \pi c / \omega_{0}$ is the wavelength of the light. This is the minimum radius of $\max \left(w_{1}, w_{2}\right)$ for a given armlength $l$, where $w_{1}$ and $w_{2}$ are the beam radii at each end, respectively. 
One needs a safety factor of $\geq 3$ for mirror radii in order to reflect most of the light power. Thus, the mirror surface area required is

$A_{1} \approx 0.03 \mathrm{~m}^{2} \times\left[\frac{\lambda}{1 \mu \mathrm{m}}\right]\left[\frac{l}{3 \mathrm{~km}}\right]$

per reflection. When all the reflection spots are (more or less) separated, one needs a mirror surface of $b A_{1} \approx 0.5 \mathrm{~m}^{2}$ $\times[L / 100 \mathrm{~km}]$. This value does not depend much on how the multiple reflections are realized (see Ref. [13] for more detail).

This limitation is an even more serious problem in a Sagnac interferometer, since its gain is poor below the lower cut-off frequency of $0.182 f_{\mathrm{z}}$. When an optical path length of $100 \mathrm{~km}$ is chosen, the lower cut-off frequency is $546 \mathrm{~Hz}$ and the peak frequency is $1.11 \mathrm{kHz}$. A longer optical path length is required if one aims at lower frequencies; but the value quoted above is already close to the limitation of the vacuum tubes for the planned large interferometers.

\section{Power recycling}

The advantage of the higher peak gain of the Sagnac response is for the most part coming from the fact that the carrier light travels through both of the arms before recombination. This means that the amount of light energy stored in the optical system is twice that of a Michelson interferometer, under the assumption that the light power hitting the beamsplitter is the same in both cases.

In practice, however, all the planned interferometric gravitational-wave detectors will employ power recycling [5] in order to achieve the required high sensitivity. As can be seen in Eq. (3), the sensitivity improves with the square root of light power (for a given interferometer). The use of power recycling effectively enhances the light power hitting the beamsplitter by forming a cavity with the interferometer as one of the cavity mirrors (see Figs. 2, 4). As we will see shortly, the power enhancement achievable by power recycling differs between the two types of interferometers.

When power recycling is optimized for the maximum power enhancement, the equivalent power enhancement factor $\mathscr{G}_{\mathrm{PR}}$ is determined by the loss of the interferometer as

$\mathscr{G}_{\mathrm{PR}}=\left(\mathscr{A}_{\mathrm{int}}+\frac{\mathscr{A}_{\mathrm{PR}}}{1-\mathscr{A}_{\mathrm{PR}}}\right)^{-1}=\frac{1}{\mathscr{A}_{\mathrm{int}}+\mathscr{A}_{\mathrm{PR}}}$

by choosing the power reflectivity $\rho_{\mathrm{PR}}^{2}$ of the power recycling mirror to be

$\rho_{\mathrm{PR}}^{2}=\left(1-\mathscr{A}_{\mathrm{int}}\right)\left(1-\mathscr{A}_{\mathrm{PR}}\right)^{2} \simeq 1-\left(\mathscr{A}_{\mathrm{int}}+2 \mathscr{A}_{\mathrm{PR}}\right)$.
Here $\mathscr{A}_{\text {int }}$ is the power loss in the interferometer and $\mathscr{A}_{\mathrm{PR}}$ is the power loss of the power recycling mirror. Since the sideband amplitude is proportional to the carrier amplitude, the sensitivity improves proportional to the square root of the power enhancement factor $\mathscr{G}_{\mathrm{PR}}$.

Due to the fact that the carrier light travels through both arms of a Sagnac interferometer, the loss in the Sagnac interferometer is expected to be twice that in a Michelson, provided that the loss is dominated by the losses in the arms. (This may not be true if the loss of the interferometer is dominated by that at the beamsplitter, e.g. by bad interference.) Thus, the power enhancement factor achievable in a Sagnac interferometer is expected to be half that in a Michelson interferometer. In the end, the amount of light energy stored in the optical system will not be different in the two cases.

This is a natural consequence of the fact that the light experiences at least one reflection from a mirror with finite loss after each transit of the armlength. Thus, the total amount of light energy $\mathscr{E}$ that can be stored in an optical system depends only on the armlength $l$, the minimum loss per reflection $\mathscr{A}_{\text {min }}$, and the injected light power $P_{0}$, as [8]

$$
\begin{aligned}
\mathscr{E}_{\max } & =P_{0} \frac{l}{c} \frac{1-\mathscr{A}_{\min }}{\mathscr{A}_{\min }} \\
& \approx 20 \mathrm{~J} \times\left[\frac{P_{0}}{50 \mathrm{~W}}\right]\left[\frac{l}{3 \mathrm{~km}}\right]\left[\frac{\mathscr{A}_{\min }}{25 \mathrm{ppm}}\right]^{-1},
\end{aligned}
$$

not depending on the configuration employed. (The quoted values are typical design parameters of planned large interferometers.)

Fig. 3 shows a comparison of the two types of interferometers, with and without power recycling. Since the effect of power recycling is equivalent to the use of a light source with higher power, the characteristic shape of each

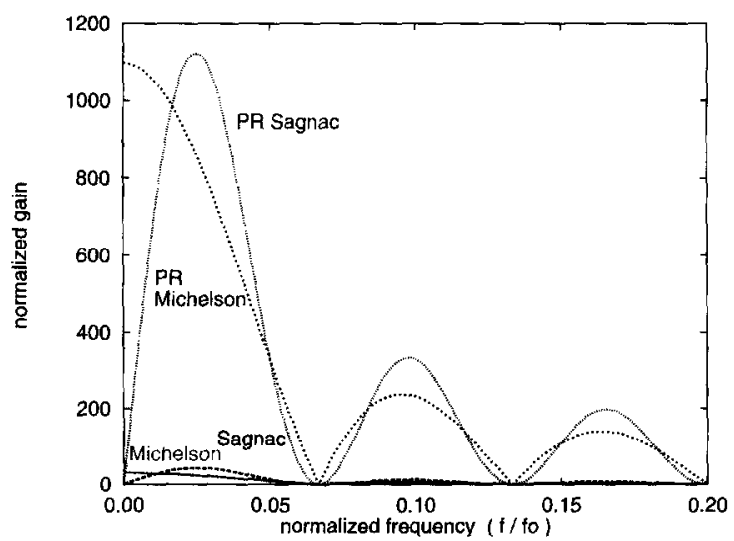

Fig. 3. Response of Michelson and Sagnac interferometers with multiple reflections in the arms $(N=30)$. Those with (upper curves) and without (lower curves) power recycling are shown. When power recycling is adopted, the peak gains of the two types of interferometers become very similar. 

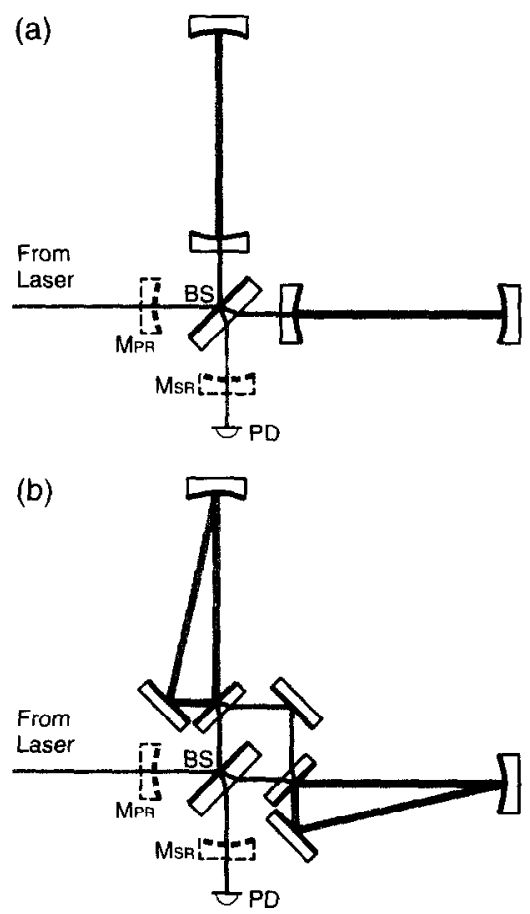

Fig. 4. Schematics of (a) Michelson and (b) Sagnac layout using an optical cavity in each arm. Positions for possible power- and signal-recycling (extraction) mirrors $\left(\mathrm{M}_{\mathrm{PR}}, \mathrm{M}_{\mathrm{SR}}\right)$ are also shown (in dashed lines).

response does not change. When power recycling is adopted, however, the overall height can be increased by a significant factor $(\sim 30)$. The peak height of the Sagnac interferometer comes very close to that of the Michelson. due to the difference in power recycling factor. In Fig. 3. $N=30$ transits in each arm are assumed.

As can be seen in Fig. 3, the improvement in gain due to power recycling will be substantial. Thus, in the following discussion we assume that power recycling is always adopted and optimized for the maximum power enhancement.

\section{Optical cavities in the arms}

In the case of Michelson interferometers. the use of an optical cavity (either Fabry-Perot type or ring type) in each arm was proposed [5] as an alternative means to realize long storage times in the arms. The same scheme can be used for Sagnac interferometers, and we consider it here (see Fig. 4).

There is a multiple interference inside a cavity which complicates the analysis. The optical response of a Fabry-
Perot cavity can be obtained through a detailed analysis [8]:

$$
G_{\mathrm{cav}}=\frac{\tau_{\mathrm{c}}}{1-\rho_{\mathrm{c}} \rho_{\mathrm{r}}} \frac{-\tau_{\mathrm{c}}}{1-\rho_{\mathrm{c}} \rho_{\mathrm{r}} \mathrm{e}^{-s t_{\mathrm{c}}}} \rho_{\mathrm{r}} \frac{\omega_{0}}{4} \frac{1-\mathrm{e}^{-s t_{\mathrm{c}}}}{s},
$$

where $\rho_{c}, \tau_{c}, \rho_{\mathrm{r}}$ represent the amplitude reflectivity and transmittance of the coupling mirror and the reflecting mirror, respectively, and $t_{c}$ is the round-trip time in the cavity $\left(t_{c}=2 l / c\right.$ for a Fabry-Perot cavity with length $\left.l\right)$. For ring-type cavities, $\rho_{c} \rho_{r}$ must be replaced by the total amplitude attenuation during a round trip.

In the case of a Michelson with a cavity in each arm. the effects on the light in the two arms are the same (with opposite polarity). Thus, the optical response is the same as that of the cavity in one arm. It turns out that the effect of the cavity is similar to that of multiple reflections: a cavity with finesse $\mathscr{F}$ and length $l$ yields similar gain and bandwidth to an effective optical path length of

$L_{\mathrm{eff}} \simeq \frac{4}{\pi} \rightrightarrows l, \quad$ or $N_{\mathrm{eff}} \simeq \frac{4}{\pi} \mathscr{F}$

using the effective number of transits (see Fig. 5).

For the Sagnac with an optical cavity in each arm, the sidebands produced in the first and the second arm must be added in a way similar to the multiple-reflection Sagnac. In this case, they are added at the coupling mirror of the second cavity. The sidebands produced in the first cavity are reflected by the second cavity, with a certain amplitude reflectivity as well as a certain phase shift upon the reflection.

This is represented by a complex reflectivity of a cavity which is given as

$\rho_{\text {cav }}(\psi)=\frac{\rho_{\mathrm{c}}-\left(1-\mathscr{A}_{\min }\right) \rho_{\mathrm{r}} \mathrm{e}^{-i \psi}}{1-\rho_{\mathrm{c}} \rho_{\mathrm{r}} \mathrm{e}^{-i \psi}}$.

Here $\psi$ is the tuning of the cavity at that frequency,

$\psi=\psi_{0}+\omega t_{\mathfrak{c}}$,

where $\psi_{0}$ is the amount of detuning (the tuning at the carrier frequency, usually set to $\psi_{0}=0$ ) and $\omega$ is the frequency offset from the carrier (positive for the upper sideband and negative for the lower sideband).

Another fact to be noted is that the carrier which enters the second cavity is that reflected by the first cavity. There is not only the amplitude loss factor but also the phase shift upon reflection. When 'over-coupled' cavities ${ }^{4}$ are used (as expected in interferometric gravitational-wave detectors), the carrier experiences a phase shift of $\pi$.

\footnotetext{
${ }^{4}$ A cavity in which the power transmittance of the coupling mirror dominates the total loss inside the cavity.
} 
Combining the above relations, the frequency response of a Sagnac interferometer with optical cavities in the arms can be written as

$G_{\mathrm{cavSag}}=\left\{\rho_{\mathrm{cav}}(0)-\rho_{\mathrm{cav}}\left(\omega t_{\mathrm{c}}\right)\right\} G_{\mathrm{cav}}$,

where both arm cavities are assumed to be resonant with the carrier frequency.

(There is actually an additional parameter, that is the path length from one arm cavity to another. It is assumed from practical considerations that this length is short compared with the arm cavity length. If there is significant distance $L_{\text {bet }}$ between the two cavities, there will be an additional factor $\exp \left\{-\mathrm{i} \omega L_{\text {bet }} / c\right\}$ in front of the second term in Eq. (19).)

Fig. 5 shows the response of (power-recycled) Michelson and Sagnac interferometers with cavities in the arms, together with the response of those with multiple-reflection arms. The finesse of the cavities is assumed to be $\approx 200$. (The multiple-reflection responses are shown for comparison, although for kilometer-class interferometers $L=N l \simeq$ $(4 / \pi) \mathscr{F} l \approx 764 \mathrm{~km} \times[l / 3 \mathrm{~km}]$ is quite unrealistic.)

In the case of Michelson-based interferometers, the use of cavities gives a peak gain similar to the equivalent multiple-reflection case but with a slightly narrower bandwidth (when compared at the $-3 \mathrm{~dB}$ points), provided that Eq. (16) is satisfied. On the other hand, the cavity response has no zeros within $f<f_{0}=c / 2 l$, resulting in a better sensitivity around the zeros in the multiple-reflection response.

In the Sagnac case, the peak gain with cavities in the arms is about 0.7 times that with multiple-reflection arms, assuming the relations in Eq. (16). In addition, the peak is located at a slightly lower frequency. These results come from combining the characteristics of a Sagnac interferometer and of cavities in the arms. As in the case of the Michelson, there are no zeros in the cavity response,

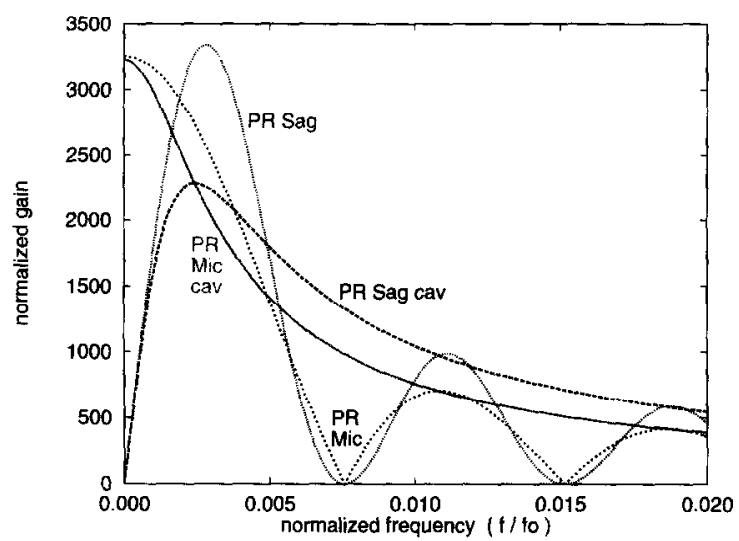

Fig. 5. Response of Michelson and Sagnac interferometers with cavities in the arms $(\mathscr{F} \approx 200)$. Power recycling is optimized. Those with multiple-reflection arms $(N=(4 / \pi) \mathscr{F} \approx 254)$ are also shown for comparison excepting the one at DC (which comes from the Sagnac characteristics). This makes the response broader when measured at the $-3 \mathrm{~dB}$ points.

It is also possible to make comparisons with conditions different from Eq. (16). Thus we do not make any definite conclusion here and postpone that to after evaluating other types of responses in the coming sections.

\section{Signal recycling}

\subsection{Principle of signal recycling}

Another scheme proposed for Michelson interferometers to improve the low frequency performance is signal recycling [6]. Its effect is to increase the peak sensitivity at the expense of narrowing the detector bandwidth. Another benefit of this scheme is that one can alter the frequency of the peak sensitivity, which is, however, in general worse than the DC case by a factor of $\sqrt{2}$ (for the same bandwidth).

The scheme of signal recycling is especially valuable for interferometers with multiple reflections in the arms, since the mirror size problem mentioned earlier practically limits the low-frequency performance of these interferometers. When the interferometer employs cavities in the arms, the additional mirror $\left(\mathrm{M}_{\mathrm{SR}}\right.$ in Fig. 4) forms a coupled cavity with the arm cavities of the interferometer $[8,11]$. This results in the effect of signal recycling or resonant sideband extraction, depending on the tuning condition of that additional mirror.

To implement signal recycling, the additional mirror ( signal recycling mirror, $\mathrm{M}_{\mathrm{SR}}$ in Figs. 2, 4) is positioned so as to form a cavity with the interferometer in a way similar to the case of power recycling, but this time at the detection port (see Figs. 2, 4). The sidebands produced by a differential modulation of the interferometer will be stored in this cavity ('signal cavity' for simplicity). Duc to the multiple interference inside this cavity, the sidebands that are resonant with this cavity are enhanced, thus improving the sensitivity. On the other hand, the sidebands that are off-resonant are suppressed by the same mechanism.

This enhancement or suppression is expressed by the signal recycling factor given as

$$
H=\frac{\tau_{\mathrm{s}}}{1-\rho_{\mathrm{s}} \rho_{\mathrm{int}} \mathrm{e}^{-i \phi}} .
$$

Here $\rho_{\text {int }}$ is the (possibly complex) amplitude reflectivity of the interferometer, $\rho_{\mathrm{S}}$ and $\tau_{\mathrm{S}}$ are the amplitude reflectivity and transmittance of the signal recycling mirror, and $\psi$ is the tuning of the signal cavity at that frequency, given by

$\psi=\psi_{0}+\omega t_{\mathrm{S}}$ 


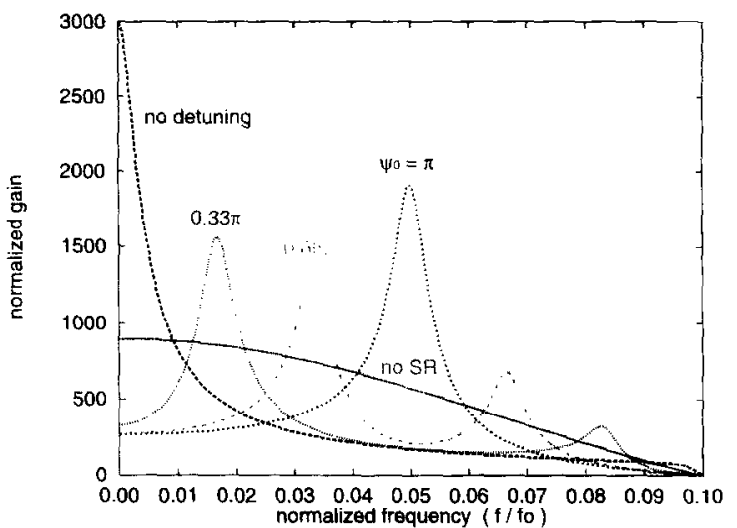

Fig. 6. Response of a power-recycled Michelson interferometer with multiple-reflection arms $(N=20)$ and a signal recycling mirror $\left(\left|\rho_{\mathrm{S}}\right|^{2} \approx 0.7\right)$. Each curve represents a different tuning condition of the signal recycling mirror. The response without the signal recycling mirror is also shown for comparison.

where $\psi_{0}$ is the amount of detuning and $t_{\mathrm{S}}$ is the round-trip time in the signal cavity.

When cavities are used in the arms, the interferometer has a frequency-dependent amplitude reflectivity as well as a frequency-dependent phase shift upon reflection. This is represented by using a complex reflectivity similar to that mentioned in Section 5.

\subsection{Signal recycling with multiple-reflection arms}

Fig. 6 shows some examples for the response of a multiple-reflection $(N=20)$ Michelson interferometer with signal recycling. When there is no detuning $\left(\psi_{0}=0\right)$, the gain peak is located at $D C$, with a higher but narrower peak than that without signal recycling. Outside this peak, the gain is lower than that without signal recycling.

When there is a finite detuning $\psi_{0}$, the response has two peaks within $0 \leq f \leq f_{z}=c / N I$ : at $\left(\psi_{0} / 2 \pi\right) f_{z}$ and $\left(1-\psi_{0} / 2 \pi\right) f_{7}$. This is the result of superimposing the responses for the upper and lower sidebands to a single (positive) frequency (see Eq. (2)). As shown in Fig. 7, one of the peaks is for the upper sideband and the other is for the lower one, the distance between the two being the

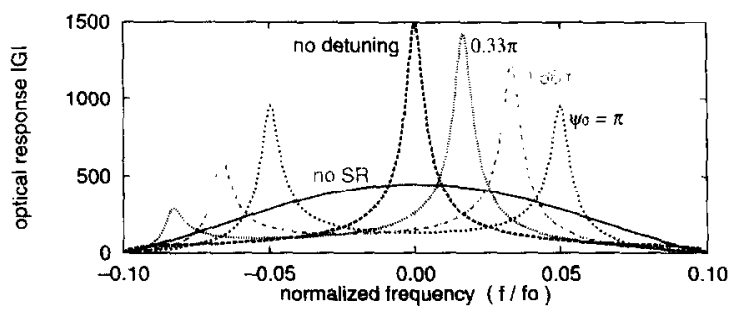

Fig. 7. The same response as Fig. 6, but shown as 'single sideband' response in the positive (upper sideband) and negative (lower sideband) frequencies.

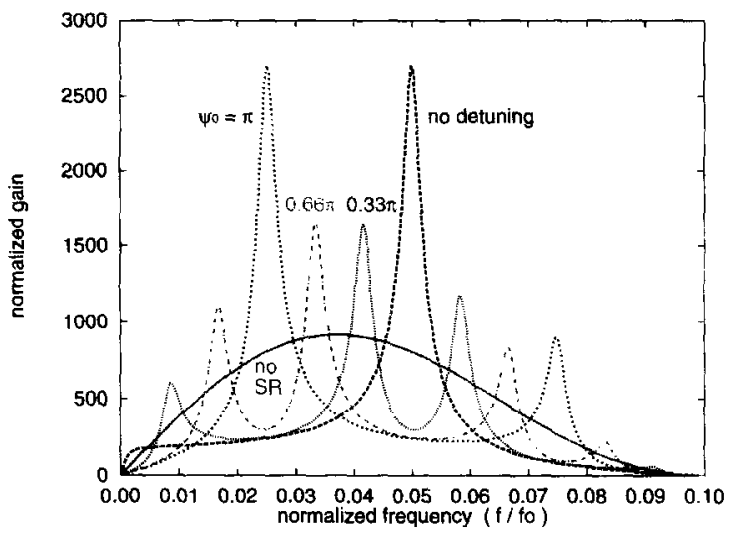

Fig. 8. Response of a power-recycled Sagnac interferometer with multiple-reflection arms $(N=20)$ and a signal recycling mirror $\left(\left|\rho_{S}\right|^{2} \approx 0.7\right)$. Each curve represents a different tuning condition of the signal recycling mirror. The response without signal recycling mirror is also shown for comparison.

FSR $^{5}$ (given by $c / N l=f_{\mathrm{z}}$ ) of the signal cavity. (When the detuning is $\psi_{0}=\pi$, the two peaks coincide and compose one single peak.)

These two peaks are higher by the same factor than the response without signal recycling. The one closer to $\mathrm{DC}$ is, however, higher than the other because the response without signal recycling is higher at that frequency. Since we are usually interested in a single frequency range, we can neglect the smaller peak and adjust the higher one (at the lower frequency) for the particular purpose.

When the same signal recycling mirror is used in different detuning conditions, even the higher peak in the detuned case is about half in height of that in the non-detuned case, whereas the width of each peak is twice that of the non-detuned case. These differences come from the fact that in the non-detuned case the two sides of a peak (located at $\omega=0$ ) are superimposed to compose one single peak.

A similar, but slightly different, effect can be obtained when signal recycling is used with Sagnac-based interferometers. Since 'one round trip' inside the signal cavity in this case includes both arms, the round-trip time is twice that in the Michelson case (assuming the optical path length in one arm to be the same in the two cases). Thus, the FSR in the signal cavity is $c / 2 N l=f_{z} / 2$, half that of the Michelson case.

Fig. 8 shows some examples for the response of a multiple-reflection $(N=20)$ Sagnac interferometer with signal recycling. When there is no detuning, a single peak appears at $f=0.5 f_{z}$, with a height and width similar to the

\footnotetext{
${ }^{5}$ The FSR (free-spectral-range) of a cavity is given by $c / L_{r}$, where $L_{\mathrm{r}}$ is the path length in a round trip.
} 
Michelson (non-detuned) case. This is not surprising knowing the result without signal recycling.

When there is finite detuning, four instead of two peaks will appear within $0 \leq f \leq f_{7}$ (though not all of them are significant). This is a consequence from the FSR being half that of the Michelson case. The peak frequencies are $\left(0.5 \pm \psi_{0} / 4 \pi\right) f_{z},\left(\psi_{0} / 4 \pi\right) f_{z}$, and $\left(1-\psi_{0} / 4 \pi\right) f_{z}$, respectively. When the detuning is $\pi$, two of the peaks coincide and compose a single peak at $f=0.25 f_{z}$, again with a height and width similar to the Michelson (and non-detuned) case.

\subsection{Signal recycling with cavity arms}

When optical cavities are used in the arms of an interferometer, its reflectivity, $\rho_{\text {int }}$ in Eq. (20), will be a frequency-dependent complex reflectivity, as mentioned earlier. In the case of a Michelson-based interferometer, it will be the complex reflectivity of the arm cavity, shown in Eq. (17). For a Sagnac-based one, the round trip of an interferometer contains the reflections from the two cavities in series. Thus, the complex reflectivity of the interferometer is the square of Eq. (17), provided the path length between the two cavities is short compared with the armlength.

Fig. 9 shows the response of a cavity Michelson with signal recycling, with different tuning conditions. When there is no detuning, an effect similar to the multiple-reflection case is obtained: a narrower but higher peak at DC. When the amount of detuning is changed, the peak frequency moves to a different frequency, and the height as well as the width of the peak varies. When the detuning is $\pi$, there is no significant peak and a flat, broad response is obtained.

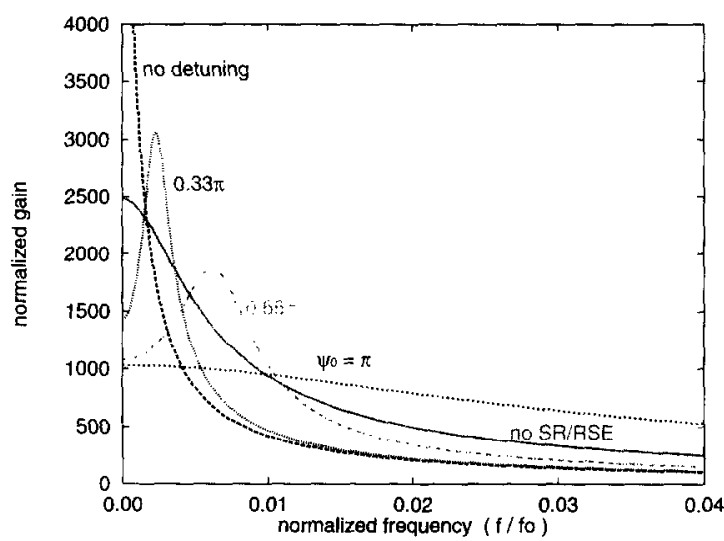

Fig. 9. Response of a power-recycled Michelson interferometer with cavity arms $(\mathscr{F} \approx 120)$ and a signal recycling mirror $\left(\left|\rho_{\mathrm{S}}\right|^{2}\right.$ $\approx 0.5$ ). Each curve represents a different tuning condition of the signal recycling mirror. The response without signal recycling mirror is also shown for comparison.

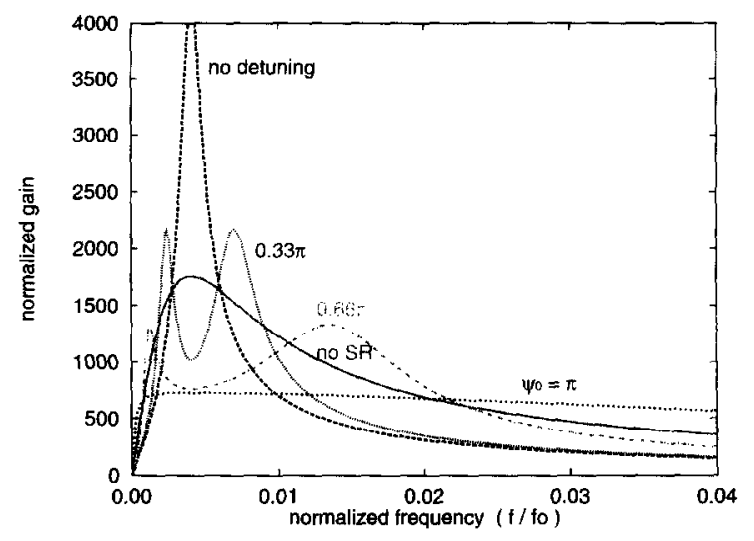

Fig. 10. Response of a power-recycled Sagnac interferometer with cavity arms $(F \approx 120)$ and a signal recycling mirror $\left(\left|\rho_{\mathrm{S}}\right|^{2} \approx 0.5\right)$. Each curve represents a different tuning condition of the signal recycling mirror. The response without signal recycling mirror is also shown for comparison.

The last case is classified as resonant sideband extraction [7,8,11], where the bandwidth of the arm cavities should be set narrower than the frequency range of interest. In any case, the response shown in Fig. 9 should be taken as examples to show the characteristics of the response in a qualitative way. The actual parameters will be chosen so that the resultant response is best adapted for the observation purpose.

The response of a cavity Sagnac with signal recycling is shown in Fig. 10. When there is no detuning, the peak height improves at the expense of a narrower bandwidth, without changing the peak frequency. When the detuning is $\pi$ radians, a flat response is obtained, similar to the resonant sideband extraction in the Michelson case.

With other tuning conditions, the peak is split into two smaller ones, at both sides of the original peak frequency. It is interesting to note that these two peaks have the same height but different widths, which are opposite to the multiple-reflection case. It may also worth mentioning that in logarithmic scale (not shown) these two peaks appear symmetric at the both sides of the original peak. However, such a response with two peaks is not expected to be very useful.

Thus, the only reasonable choice of detuning is $\psi_{0}=0$ or $\pi$. In the latter case, the lower cut-off frequency becomes so low that the response is not different from other broad band responses (e.g. resonant sideband extraction). The non-detuned response has a high peak at a frequency that is determined by the finesse of the arm cavity. Again, a similar response can be obtained also by other configurations (e.g. signal recycling). Considering its complex layout, there is no reason to prefer the cavity Sagnac with signal recycling. Thus, we neglect this configuration in the following discussion. 


\section{Choice of parameters}

\section{J. Concerning shot noise}

With the above results, we consider the application of these interferometers for gravitational-wave detection. Although we do not know the exact waveform we should expect, the desired response for typical applications would be: a relatively broad $(\sim 1 \mathrm{kHz})$ response for burst-like signals, one optimized at low frequency $(\sim 100 \mathrm{~Hz})$ for 'chirp' signals from coalescing binary stars, and a narrowed one at a well-defined frequency for the signal from a known pulsar.

For a broad-band type response with multiple-reflection arms, the biggest problem is the mirror size required. Even with $100 \mathrm{~km}$ path length, the sensitivity of a Sagnac interferometer below $500 \mathrm{~Hz}$ is poor, as mentioned earlier. In the case of a Michelson-based interferometer, the lessthan-optimum path length can be compensated by using (non-detuned) signal recycling. For a Sagnac-based one, however, one absolutely needs to realize a long path length in the arms in order to bring the lower cut-off frequency $0.182 c / L$ below the frequency range of interest.

One straightforward way to make a longer path length with the given mirror size is the use of a light source with shorter wavelength. The area of the mirror surface required is proportional to the wavelength of the light, as can be seen from Eq. (11). On the other hand, the light power available and the losses of optical components depend on the wavelengths.

The shot-noise-limited sensitivity to gravitational waves (for a fixed detector bandwidth) is proportional to $\sqrt{\lambda / \eta \mathscr{E}}$ where $\boldsymbol{\eta}$ is the efficiency of the photo-detector, and $\mathscr{E}$ is the amount of light energy stored $[8,12]$. Assuming that power recycling is optimized and that the loss of the interferometer is dominated by the losses in the arms, the amount of light energy stored will be close to that in Eq. (14). Thus, the 'figure of merit' in the choice of the wavelength is

$$
\frac{\eta(\lambda) P_{0}(\lambda)}{\lambda \otimes_{\min }(\lambda)}
$$

The light source must be chosen to maximize this quantity, as far as the shot noise is concerned. It is possible, however, that other noise sources are more important in the choice, once the light power available becomes high enough.

When cavities are used in the arms, the peak sensitivity of a Sagnac-based interferometer is worse than that of a Michelson-based one, if both use the same arm cavities. It is possible to use a higher finesse for the Sagnac in order to achieve a higher peak sensitivity. When one chooses the finesse for the Sagnac twice that for the Michelson, a response with height and width similar to those of Michelson one is obtained (see Fig. 11).
In addition, the peak is located not at DC but slightly away from it, depending on the finesse of the arm cavities. This is advantageous, since the sensitivity at the lowfrequency end $(f \leq 50 \mathrm{~Hz}$ ) will anyway be dominated by noise sources other than shot noise (seismic, pendulum thermal, gravity gradient). When the lower cut-off frequency of the Sagnac response is close to this lowfrequency end, one can make use of the whole bandwidth of the detector. Since the finesse of the arm cavities determines the bandwidth as well as the lower cut-off frequency of the detector (i.e. they are not independent), a reasonable choice of the finesse results in a response with a peak around 100 to $200 \mathrm{~Hz}$. This is quite suitable for the 'low frequency' purpose mentioned earlier.

As a drawback, one is likely forced to use ring cavities rather than Fabry-Perot cavities in a Sagnac interferometer in order to separate incoming and outgoing beams. This means more surfaces and thus more losses in the round trip of an arm, which results in less light energy stored in the arms. (This effect is not included in Fig. 11.)

Fig. 11 shows some of the examples optimized for 100 to $200 \mathrm{~Hz}$, assuming an armlength of $3 \mathrm{~km}$. The four configurations shown are: a Michelson with cavity arms (the 'standard' configuration), a Sagnac with cavity arms. a four-bounce Michelson with signal recycling, and resonant sideband extraction. As discussed above, the Sagnac one is somewhat better than the standard configuration in the frequency range of interest. However, a similar response can also be obtained by using either signal recycling or resonant sideband extraction, which may be somewhat advantageous both in sensitivity and in flexibility.

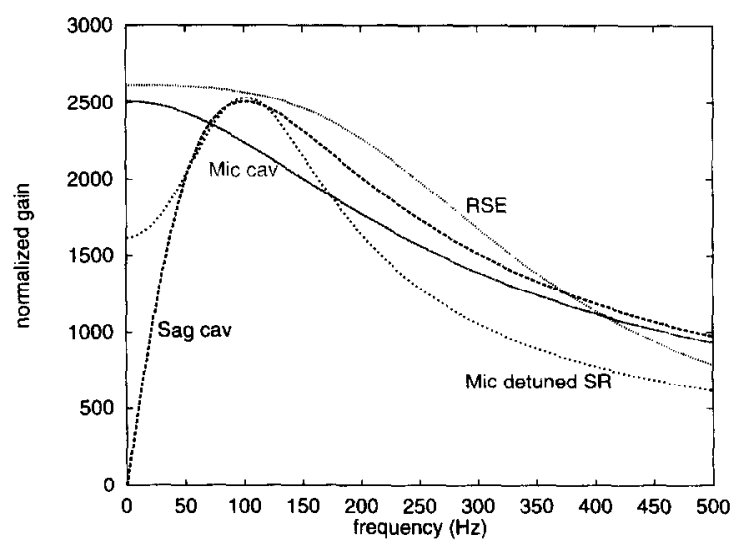

Fig. 11. Response optimized for 100 to $200 \mathrm{~Hz}$, assuming $3 \mathrm{~km}$ armlength. The curves are: a Michelson with cavity arms $(F \approx$ $125)$, a Sagnac with cavity arms $(\mathscr{F} \approx 250)$, a multi-reflection Michelson with detuned signal recycling $\left(N=4, \rho_{\mathrm{S}}^{2} \approx 0.977\right.$ and $\psi_{0} \approx 0.23 \mathrm{rad}$ ), and resonant sideband extraction (arm cavity $\mathscr{F} \approx$ 5000 and $\rho_{S}^{2} \approx 0.9$, the signal extraction mirror is placed $3 \mathrm{~km}$ away from the beamsplitter and zero detuning). It might worth mentioning that a Michelson with cavity arms shows better sensitivity than others at frequencies higher than those shown here. 
A narrow-band response can be obtained from a Sagnac-based interferometer by using signal recycling. The detuning must, however, be chosen to be either 0 or $\pi$ to achieve a high peak. Then the storage time in the arms (set either by multiple reflections or by cavities) uniquely determines the peak frequency. It is possible to have a peak at other frequencies by using other detuning conditions, but the peak height in this case is somewhat lower than in the optimum case. In any case, the storage time in the arms must be chosen reasonably long, either by multiple reflections or by cavities, to have a peak at a relatively low frequency.

\subsection{Other issues}

One of the advantages of a Sagnac-based interferometer is its alleged insensitivity to low-frequency fluctuations of the arm lengths, which eliminates the necessity of controlling the mirror positions [9]. This is true, but only for non-recycled, multiple-reflection ones. Once cavities are used either in the arms or for power- or signal-recycling, the relative position must be controlled precisely by using active feedback control (although the number of degrees to be controlled may decrease by one). The control signal is in general obtained optically from the interferometer. The frequency response of a Sagnac-based interferometer is, however, not as simple as that of a Michelson-based one and may complicate the control task.

Another virtue of a non-recycled, multiple-reflection Sagnac interferometer is that it is insensitive to variations of light frequency, which makes it possible to use light with reduced coherence length [9] in order to overcome the scattered-light problem [14]. Again, this meets a difficulty once cavities are used. Although it may in principle be possible to modulate the cavity lengths synchronously to the light frequency, it will be very difficult to avoid the coupling to the measurement bandwidth.

The lack of power recycling can, of course, be compensated by the use of a higher-power light source. Once the light power is strong enough so that the sensitivity is determined by other sources rather than shot noise, there is no necessity for power recycling. The typical power recycling factor planned is 30 to 100 (depending on the storage time in the arms). This means that one would require $\gg 1 \mathrm{~kW}$ laser with quite high stability in order to make an interferometer of the desired sensitivity without power recycling.

\section{Conclusion}

The frequency response of Sagnac-based interferometers with multiple reflections or cavity arms is evaluated and compared with that of Michelson-based ones. The effects expected from power and signal recycling are also considered. In order to achieve a good sensitivity at a relatively low frequency $(\leqslant 1 \mathrm{kHz})$, a reasonably long storage time in one arm (either by multiple reflections or by a cavity) is required. In the Sagnac case, the use of the signal recycling scheme cannot help the less-than-optimum storage time in the arms. The mirror size problem may limit the application of the multiple-reflection choice, unless a suitable light source with a short wavelength becomes available.

The most promising configuration based on a Sagnac interferometer will be the one with cavity arms, optimized for relatively low frequencies $(100-300 \mathrm{~Hz})$. In general, however, the authors do not see particular advantages in Sagnac-based interferometers over Michelson-based ones for the purpose of gravitational-wave detection.

\section{References}

[1] A. Abramovici, W.E. Althouse, R.W.P. Drever, Y. Gürsel, S. Kawamura, F.J. Raab, D. Shoemaker, L. Sievers, R.E. Spero, K.S. Thorne, R.E. Vogt, R. Weiss, S. Whitcomb and M.E. Zucker, Science 256 (1992) 325.

[2] C. Bradaschia, R. Del Fabbro, A. Di Virgilio, A. Giazotto, H. Kautzky, V. Montelatici, D. Passuello, A. Brillet, O. Cregut, P. Hello, C.N. Man, P.T. Manh, A. Marraud, D. Shoemaker, J.Y. Vinet, F. Barone, L. Di Fiore, L. Milano, G. Russo, J.M. Aguirregabiria, H. Bel, J.P. Duruisseau, G. Le Denmat, Ph. Tourrenc, M. Capozzi, M. Longo, M. Lops, I. Pinto, G. Rotoli, T. Damour, S. Bonazzola, J.A. Marck, Y. Gourghoulon, L.E. Holloway, F. Fuligni, V. Iafolla and G. Natale, Nucl. Instrum. Methods Phys. Res. A 289 (1990) 518.

[3] K. Danzmann, H. Lück, A. Rüdiger, R. Schilling, M. Schrempel, W. Winkler, J. Hough, G.P. Newton, N.A. Robertson, H. Ward, A.M. Campbell, J.E. Logan, D.I. Robertson, K.A. Strain, J.R.J. Bennett, V. Kose, M. Kühne, B.F. Schutz, D. Nicholson, J. Shuttleworth, H. Welling, P. Aufmuth, R. Rinkeff, A. Tünnermann and B. Willke, GEO 600 - A $600 \mathrm{~m}$ Laser Interferometric Gravitational Wave Antenna, in: First Edoardo Amaldi Conference on Gravitational Wave Experiments, eds. E. Coccia, G. Pizzella and F. Ronga (World Scientific, Singapore, 1995) pp. 100-111.

[4] R. Weiss, MIT Quarterly Progress Report (Research Laboratory of Electronics) 105 (1972) 54.

[5] R.W.P. Drever, J. Hough, A.J. Munley, S.-A. Lee, R. Spero, S.E. Whitcomb, H. Ward, G.M. Ford, M. Hereld, N.A. Robertson, I. Kerr, J.R. Pugh, G.P. Newton, B. Meers, E.D. Brook III and Y. Gürsel, Gravitational wave detectors using laser interferometers and optical cavities: Ideas, principles and prospects, in: Quantum Optics, Experimental Gravitation and Measurement Theory, eds. P. Meystre and M.O. Scully (Plenum Press, New York, 1983) pp. 503-514.

[6] B.J. Meers, Phys. Rev. D 38 (1988) 2317.

[7] J. Mizuno, K.A. Strain, P.G. Nelson, J.M. Chen, R. Schilling, A. Rüdiger, W. Winkler and K. Danzmann, Phys. Lett. A 175 (1993) 273.

[8] J. Mizuno, Internal report MPQ 203 (1995).

[9] K.X. Sun, M.M. Fejer, E. Gustafson and R.L. Byer, Phys. Rev. Lett. 76 (1996) 3053. 
[10] J.-Y. Vinet, B. Meers, C.N. Man and A. Brillet, Phys. Rev. D 38 (1988) 433 .

[11] G. Heinzel, J. Mizuno, R. Schilling. W. Winkler, A. Rüdiger and K. Danzmann, Phys. Lett. A 217 (1996) 305.

[12] J. Mizuno et al., paper in preparation (1996).
[13] W. Winkler, A Michelson interferometer using delay lines, in: The Detection of Gravitational Waves, ed. D.G. Blair (Cambridge University Press. Cambridge, 1991) pp. 269-305.

[14] R. Schilling, L. Schnupp, W. Winkler, H. Billing, K. Maischberger and A. Ruidiger. J. Phys. E 14 (1981) 65. 